\title{
Does vancomycin resistance increase mortality in Enterococcus faecium bacteraemia after orthotopic liver transplantation? A retrospective study
}

S. Dubler ${ }^{1 *}$ (D, M. Lenz ${ }^{1,2}$, S. Zimmermann ${ }^{3}$, D. C. Richter ${ }^{1}$, K. H. Weiss ${ }^{4}$, A. Mehrabi $^{5}$, M. Mieth ${ }^{5}$, T. Bruckner ${ }^{6}$, M. A. Weigand ${ }^{1}$, T. Brenner ${ }^{1+}$ and A. Heininger ${ }^{7 \dagger}$

\begin{abstract}
Background: The relevance of vancomycin resistance in enterococcal blood stream infections (BSI) is still controversial. Aim of this study was to outline the effect of vancomycin resistance of Enterococcus faecium on the outcome of patients with BSI after orthotopic liver transplantation (OLT).

Methods: The outcome of OLT recipients developing BSI with vancomycin-resistant (VRE) versus vancomycinsusceptible Enterococcus faecium (VSE) was compared based on data extraction from medical records. Multivariate regression analyses identified risk factors for mortality and unfavourable outcomes (defined as death or prolonged intensive care stay) after 30 and 90 days.

Results: Mortality was similar between VRE- $(n=39)$ and VSE- $(n=138)$ group after $30(p=0.44)$ or 90 days ( $p=$ 0.39). Comparable results occurred regarding unfavourable outcomes. Mean SOFA $A_{\text {Non-GCS }}$ score during the 7-dayperiod before BSI onset was the independent predictor for mortality at both timepoints (HR 1.32; Cl 1.14-1.53; and HR 1.18; Cl 1.08-1.28). Timely appropriate antibiotic therapy, recent ICU stay and vancomycin resistance did not affect outcome after adjusting for confounders.
\end{abstract}

Conclusion: Vancomycin resistance did not influence outcome among patients with Enterococcus faecium bacteraemia after OLT. Only underlying severity of disease predicted poor outcome among this homogenous patient population.

Trial registration: This study was registered at the German clinical trials register (DRKS-ID: DRKS00013285).

Keywords: Enterococci, Bacteraemia, Mortality, Vancomycin resistance, Liver transplantation

\section{Background}

For decades, enterococci were considered to be harmless inhabitants of the human gastrointestinal tract, only rarely causing opportunistic infections in critically ill patients. Currently, they have been studied to be among leading pathogens of nosocomial infections, and thus they are a major international health burden [1-3]. Enterococci are one of the most common isolated species causing

\footnotetext{
* Correspondence: Simon.Dubler@med.uni-heidelberg.de

${ }^{\dagger} \mathrm{T}$. Brenner and A. Heininger contributed equally to this work.

'Department of Anaesthesiology, Heidelberg University Hospital, Im

Neuenheimer Feld 110, D-69120 Heidelberg, Germany

Full list of author information is available at the end of the article
}

nosocomial blood stream infections (BSI) in intensive care units, ranking second in the United States [4] and third in European intensive care units [5]. Crude mortality rates are between 20 and $50 \%$ in these patient populations $[2,6,7]$.

Treatment of enterococcal blood stream infections has become more difficult due to the increase of multidrug resistance. Prevalence of vancomycin-resistant enterococci (VRE) has increased worldwide. In a US study, up to $80 \%$ of Enterococcus faecium isolates proved to be vancomycinresistant [8]. The proportion of vancomycin resistance in enterococcal BSI rose from $5.9 \%$ in 2007 to $16.7 \%$ in 2016 [9] in German ICUs. The independent impact of this increasing vancomycin resistance on patients' outcome is still

(c) The Author(s). 2020 Open Access This article is distributed under the terms of the Creative Commons Attribution 4.0 International License (http://creativecommons.org/licenses/by/4.0/), which permits unrestricted use, distribution, and reproduction in any medium, provided you give appropriate credit to the original author(s) and the source, provide a link to the Creative Commons license, and indicate if changes were made. The Creative Commons Public Domain Dedication waiver (http://creativecommons.org/publicdomain/zero/1.0/) applies to the data made available in this article, unless otherwise stated. 
a matter of debate. Studies show conflicting results in terms of mortality of patients with VRE bacteraemia compared to cases caused by vancomycin-susceptible enterococci (VSE). Previous studies and systematic reviews were conducted prior to the availability of effective antibiotics against VRE [10-12]. Moreover, studies often included heterogeneous patient populations and did not adjust for disease severity [13]. Finally, most studies that compared outcomes between patients suffering from infections with multidrugresistant pathogens and those with susceptible microorganisms failed to consider important confounders [13, 14].

Patients after solid organ transplantation are at increased risk for nosocomial infections due to multidrug-resistant bacteria [15] and particularly to VRE bacteraemia $[10,16,17]$. Mortality rates up to $46 \%$ in the context of VRE bacteraemia have been described in these patients [10]. However, investigations to outline the independent effect of VRE on the outcome of patients after orthotopic liver transplantation (OLT) are lacking. Thus, we performed a retrospective data analysis in our liver transplantation centre of all patients, who developed BSI after OLT due to vancomycin-resistant versus vancomycin-susceptible $E$. faecium isolates within an 11-year observation period.

\section{Methods}

\section{Study design and population}

This study was conducted as a monocentric, retrospective study at the University Hospital Heidelberg (Germany). It was approved by the local ethics committee of the Medical Faculty of the University of Heidelberg (S-407/2017) and was registered at the German clinical trials register (DRKS-ID: DRKS00013285). Adult liver transplant recipients having one or more positive blood culture with $E$. faecium from January 1st, 2006 to December 31st, 2016 were eligible. The treatment of patients was based on the Heidelberger manual of OLT. This includes a standardised immunosuppression regimen consisting of corticosteroids, calcineurin inhibitors (tacrolimus or ciclosporin) and mycophenolat-mofetil (MMF). The exclusion criteria were: patients aged $<18$ years, BSI occurring prior to OLT, BSI with both, vancomycin-resistant and vancomycin-susceptible E. faecium within the same 90- days period, patients with best supportive care and death $<48 \mathrm{~h}$ after BSI and patients with missing relevant data. Fig. 1 summarizes inclusion and exclusion criteria.

\section{Patient data}

All patients of the University Hospital Heidelberg, Germany, with VRE or VSE bacteraemia during the study period were identified using the Swisslab microbiological laboratory database (Swisslab DITS GmbH, Berlin, Germany). Subsequently, a trained researcher screened the electronic medical records $\left(\mathrm{ISH}^{\circ}\right.$, SAP, Walldorf, Germany) of all VRE and VSE-bacteraemia patients, whether they had had a liver transplantation before enterococcal BSI emerged.

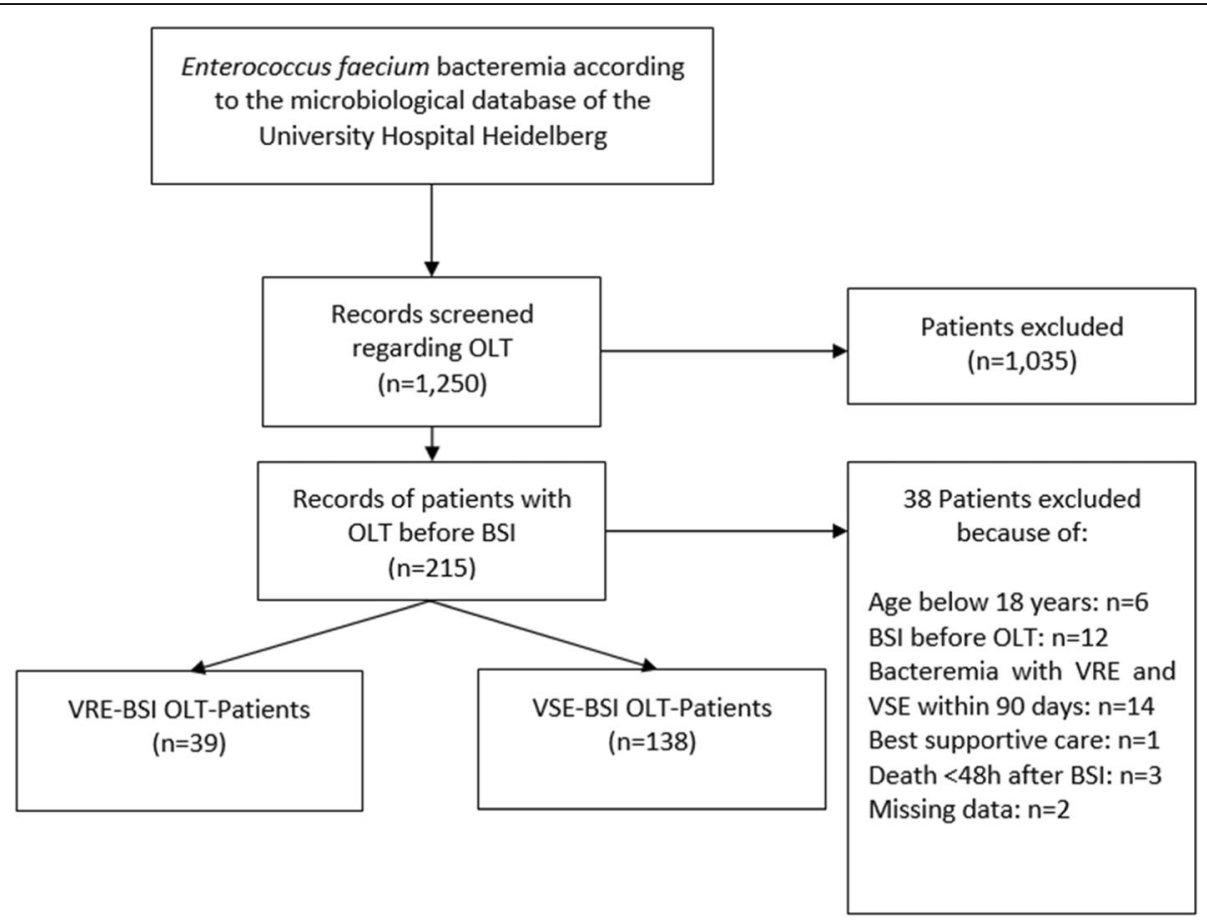

Fig. 1 Flow diagram for this study. Abbreviations: OLT, orthotopic liver transplantation; BSI, blood stream infection; VRE, vancomycin-resistant Enterococcus faecium; VSE, vancomycin-susceptible E. faecium 


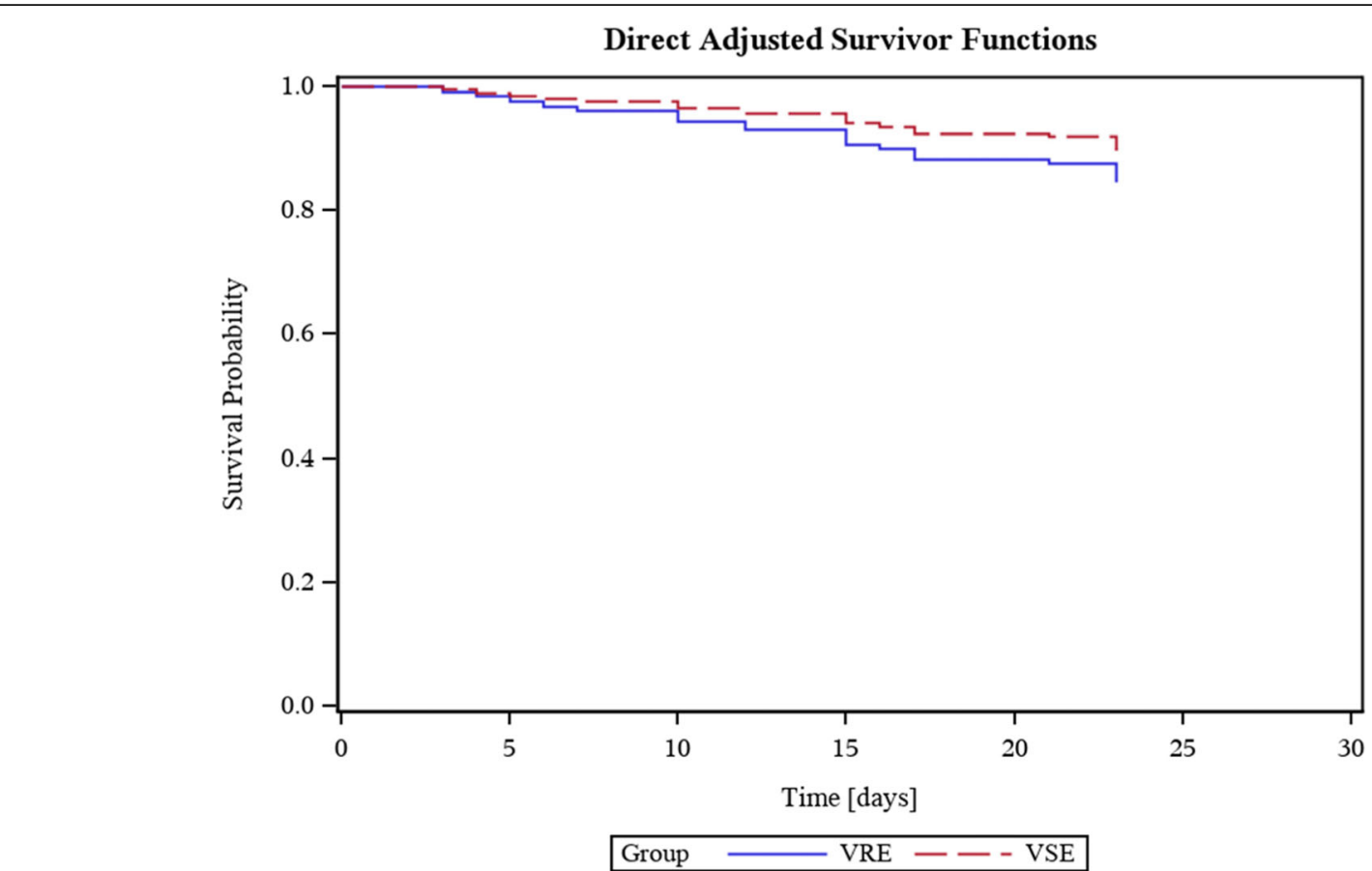

Fig. 2 Kaplan-Meier survival curves after bloodstream infections with vancomycin-resistant $E$. faecium (VRE) versus vancomycin-susceptible $E$. faecium (VSE) following orthotopic liver transplantation (OLT). Data were adjusted for confounders

The following data were extracted: demographics, Model for End-stage Liver Disease (MELD) score prior to the most recent liver transplantation before BSI, length of hospital stay pre- and post-BSI onset (ICU, ward, rehabilitation centre, at home), antibiotic therapy, surgical or other interventions, number of re-transplantations and control of the infectious focus (Table 1). To assess the acute status of illness, the sequential organ failure assessment (SOFA) score [18] was calculated. Since assessment and documentation of the Glasgow Coma Score (GCS) in ICU patients is frequently uncertain and/or incomplete, we defined a modified SOFA $\mathrm{A}_{\mathrm{Non}-\mathrm{GCS}}$ score for our data analyses, excluding the item GCS $[19,20]$. SOFA ${ }_{\text {Non-GCS }}$ scores were assessed during the 7-day period before BSI onset.

To control for potentially confounding factors, four variables beyond vancomycin resistance that may mirror the acute status of illness before BSI onset and adequacy of treatment were included in a logistic regression model for multivariate analysis of mortality as well as for the combined endpoint of an unfavourable outcome as defined below. For this aim, we applied the following items: mean SOFA $\mathrm{Non}_{\mathrm{NCCS}}$ score and number of days spent in the ICU within 7 days prior to enterococcal bacteraemia (from day -7 to day -1 ), definitive elimination of the BSI-causing infectious focus and adequacy of antibiotic treatment within $48 \mathrm{~h}$ post-BSI onset.

\section{Outcomes}

Primary endpoint was mortality (from any cause) on days 30 and 90 post-BSI onset. As secondary endpoint, we classified the outcome of patients as favourable, i.e., stay at home, on the ward or in a rehabilitation centre, versus unfavourable, i.e., death or persistent stay in the ICU, at days 30 and 90 post-BSI onset.

\section{Microbiology}

Microbial growth of blood culture bottles (BACTEC FX Aerobic/F, Anaerobic/F, BD Diagnostics, Heidelberg, Germany) was detected by the BACTEC FX ${ }^{\circ}$ automated blood culture system (BD Diagnostics, Heidelberg, Germany) and subsequently confirmed by Gram staining. Positive cultures were worked up according to approved in-hospital standard techniques. Real-time PCR for vanA and vanB genes was performed for all positive blood culture bottles with Gram-positive cocci in the smear stain as described previously. Therefore, glycopeptide resistance was usually detected on the day the blood culture signalled positive [21].

\section{Definitions}

The term enterococcal BSI was restricted to the isolation of $E$. faecium in at least one blood culture taken for clinical diagnostics. Days to appropriate antibiotic therapy was defined as period from the sampling time of the first blood culture indicating $E$. faecium to the start of at least one intravenously applied antimicrobial substance with proven efficacy (according to susceptibility testing of the blood culture isolate). Empiric antibiotic therapy was defined as the start of an antibiotic therapy before definitive results of susceptibility testing were available. Targeted 
Table 1 Baseline characteristics of subjects included in this study

\begin{tabular}{|c|c|c|c|}
\hline & $\begin{array}{l}\text { VRE-BSI } \\
(n=39)\end{array}$ & $\begin{array}{l}\text { VSE-BSI } \\
(n=138)\end{array}$ & $p$ \\
\hline Age [Years] & $53.7 \pm 10.9$ & $52.2 \pm 10.7$ & 0.408 \\
\hline Females & $11(28.2)$ & $40(29.0)$ & 0.924 \\
\hline Re-OLT before BSI onset? & & & 0.391 \\
\hline No & $18(46.2)$ & $60(43.5)$ & \\
\hline One & $21(53.9)$ & $68(49.3)$ & \\
\hline Two & $0(0)$ & $7(5.1)$ & \\
\hline Three & $0(0)$ & $3(2.2)$ & \\
\hline MELD Score last OLT* & $27.4 \pm 10.2$ & $22.9 \pm 10.8$ & 0.019 \\
\hline \multicolumn{4}{|l|}{ Interventions (d-30 until BSI onset) to: } \\
\hline increase arterial perfusion of the liver & $0.6 \pm 0.9$ & $0.3 \pm 0.7$ & 0.023 \\
\hline abscess drainage & $0.2 \pm 0.5$ & $0.2 \pm 0.6$ & 0.927 \\
\hline biliary drainage & $0.5 \pm 1.1$ & $0.8 \pm 1.2$ & 0.251 \\
\hline Laparotomies & $0.4 \pm 0.7$ & $0.5 \pm 1.0$ & 0.741 \\
\hline Total interventions (d-30 until BSI onset) & $2.3 \pm 1.8$ & $2.5 \pm 1.8$ & 0.612 \\
\hline Days from last LTX until BSI** & 25 [1-9883] & $21[1-4423]$ & 0.719 \\
\hline Mean SOFANon-GcS (d-7 until d-1 before BSI) *** & $8.1 \pm 4.6$ & $7.4 \pm 5.1$ & 0.373 \\
\hline Days in ICU before BSI onset (within 7 days before BSI) & $6.1 \pm 3.0$ & $5.1 \pm 3.5$ & 0.050 \\
\hline Days on ward before BSI onset (within 7 days before BSI) & $1.6 \pm 2.6$ & $2.2 \pm 3.1$ & 0.204 \\
\hline Days at home before BSI onset (within 7 days before BSI) & $0.3 \pm 1.4$ & $0.7 \pm 1.8$ & 0.057 \\
\hline
\end{tabular}

Data are presented as $\mathrm{n}(\%)$, mean \pm standard deviation.

* $n=176$ (1 missing)

**Values displayed as median [range]

*** $n=172$ (5 missing)

Abbreviations: VRE vancomycin-resistant Enterococcus faecium, VSE vancomycin-susceptible E. faecium, OLT orthotopic liver transplantation, ICU intensive care unit, $B S /$ bloodstream infection

antibiotic therapy was treatment given after receipt of antimicrobial susceptibility testing results. Definitive infectious focus elimination was defined as procedures resulting in a complete removal of persisting infectious clusters (e.g. cholecystectomy in the case of acute cholecystitis), differentiated from procedures for focus control (e.g. drainages for the treatment of insufficiencies of the biliodigestive anastomosis) [22, 23]. Two examiners independently reviewed the complete medical records of the patients and classified whether or not definitive infectious focus elimination was achieved. Polymicrobial bacteraemia was defined as isolation of more than one pathogen in the same blood culture.

Interventions on the biliary system were defined as percutaneous transhepatic cholangiodrainage (PTCD), endoscopic retrograde cholangiopancreatography (ERCP) and other interventions concerning the biliodigestive anastomosis (BDA) or biliary system, e.g., stenting of the bile duct. Interventions to increase liver perfusion included dilatation of liver arteria(s), stenting of liver arteria(s), catheter for lysis or infusion therapy of vasodilating drugs, coiling of aneurysm(s) and coiling of arteria lienalis.

\section{Data analysis}

The data were stored in an EXCEL file (Microsoft', Redmond, WA, USA) and then imported into SAS 9.4Win for analysis (SAS Institute Inc., Cary, NC, USA). Findings were reported using the mean and standard deviation for continuous data and absolute and relative frequencies for categorical variables. Potential differences between the groups were calculated using the MannWhitney U-test or chi-square test, as appropriate. Kaplan Meier curves and corresponding log-rank tests were used to compare the course of survival. Cox-regression and binary logistic regression models were used to identify possible prognostic factors for mortality [Hazard Ratio (HR)] and unfavourable outcomes [Odds Ratio (OR)], respectively. For all analyses, a two-sided $p$ value less than 0.05 was considered statistically significant. The reported $p$ values are descriptive due to the explorative nature of the study.

\section{Results}

During the 11-year observation period, we identified a total of 1250 patients with E. faecium BSI in our study 
centre; 177 of them were adult liver transplant recipients and were included in the final analysis (Fig. 1). Liver transplantations in these patients were performed between 1989 and 2016. In 39 of the 177 cases (21.5\%), the E. faecium isolates were VRE, whereas 138/177 (78.5\%) were VSE cases. The baseline characteristics of the patients included are presented in Table 1 . In both groups, the majority of patients were male. Age and MELD scores at OLT were comparable between the VRE and VSE group.

There was no difference between groups regarding median time from last OLT to first enterococcal BSI or meanSOFANon-GCS scores from day -7 to day -1 before BSI. During the week prior to BSI onset, VRE patients tended to spend more time on the ICU than VSE patients [ $6.1 \pm 3.0$ days versus $5.1 \pm 3.5$ days for VRE and VSE patients in mean, respectively, $(p=0.05)]$. However, the average number (mean) of interventions per patient to increase the arterial perfusion of the graft within 30 days before BSI was higher in VRE than in VSE patients. The proportion of BSI caused by more than one pathogen was low (17 of 177 cases) and similar in both groups $(p=0.29)$. See Additional file 1: Table S1 for a detailed list of co-pathogens. The by far most often observed focus of BSI was an intraabdominal infection; whereas other infection sites (such as urinary tract infections, primary blood stream infections, infections of unknown origin, others) occurred very rarely in the presented cohort of patients undergoing OLT (Additional file 1 Table S6 and Additional file 1: Table S7).

In the VRE and the VSE group, 6 of 39 (16.2\%) and 22 of 138 (17.3\%) patients, respectively, were treated empirically with effective antibiotics before receipt of the microbiological result $(p=0.88)$. Five of these six VREpatients empirically treated with linezolid were known to be carrier of VRE. For targeted antibiotic therapy in the VRE group, linezolid was most frequently prescribed [31/39 patients (79.5\%)], followed by tigecycline given in 6 of 39 (15.4\%) patients. Vancomycin was the most applied antibiotic in the VSE group [88/138 (63.8\%)], followed by linezolid [31/138 (22.5\%)]. Details are given in Additional file 1: Table S2. Adequate antibiotic treatment according to the susceptibility testing of the detected E. faecium isolate within $48 \mathrm{~h}$ post-BSI onset was established in 21 of $39(53.9 \%)$ patients in the VRE group and 83 of $138(60.1 \%)$ patients in the VSE group $(p=0.48)$. All patients who did receive antibiotic therapy during the study period were treated with an appropriate agent according to resistance testing. See Additional file 1: Table S2 for details on antibiotic therapy. Infectious source control procedures were performed in all patients; however, definitive elimination of the infectious focus was achieved within 30 days after BSI onset in only 9 of $39(23.1 \%)$ patients in the VRE group and 37 of 138 (26.8\%) patients in the VSE group $(p=0.64)$. The number of performed interventions from BSI onset until day 30 did not differ significantly between the two study groups $(p=0.20)$; more details are presented in Additional file 1 Table S3.

Table 2 presents the unadjusted outcomes after VREversus VSE-BSI in our OLT recipients. The primary outcome did not differ between both groups with a crude mortality rate 30 days after first enterococcal bacteraemia of $15.4 \%(6 / 39)$ in the VRE group and 10.9\% (15/ $138)$ in the VSE group $(p=0.44)$. See Fig. 2 for KaplanMeier survival curves. Similarly, after 90 days, 15 of 39 (38.5\%) VRE patients and 43 of 138 (31.2\%) VSE patients had died $(p=0.39)$.

Even when potential confounders mirroring the severity of illness before BSI onset (day -7 to day -1 ) were

Table 2 Unadjusted outcome analysis of patients with vancomycin-resistant Enterococcus faecium (VRE) versus vancomycinsusceptible E. faecium (VSE) bacteraemia

\begin{tabular}{|c|c|c|c|c|c|}
\hline Variables & $\mathrm{n}$ & $\begin{array}{l}\text { All patients } \\
n=177\end{array}$ & $\begin{array}{l}\text { VRE } \\
n=39\end{array}$ & $\begin{array}{l}\text { VSE } \\
n=138\end{array}$ & $p$ \\
\hline \multicolumn{6}{|l|}{ 30-day Endpoints } \\
\hline Favourable Outcome & $175^{*}$ & 95/175 (54.3) & $19 / 39(48.7)$ & $76 / 136(55.9)$ & 0.429 \\
\hline Crude mortality & 177 & 21/177 (11.9) & 6/39 (15.4) & 15/138 (10.9) & 0.441 \\
\hline \multicolumn{6}{|l|}{ 90-day Endpoints } \\
\hline Favourable Outcome & $172^{* *}$ & $91 / 172(52.9)$ & 20/39 (51.3) & $71 / 133(53.4)$ & 0.817 \\
\hline Crude Mortality & 177 & $58 / 177(32.8)$ & $15 / 39(38.5)$ & $43 / 138(31.2)$ & 0.391 \\
\hline Length of hospital stay until day 30 [days] & 177 & & $25.9 \pm 8.5$ & $24.9 \pm 7.7$ & 0.160 \\
\hline Stay in ICU until day 30 [days] & 177 & & $15.3 \pm 12.3$ & $13.5 \pm 12.0$ & 0.435 \\
\hline Ward stay until day 30 [days] & $156^{* * *}$ & & $12.6 \pm 12.1$ & $12.8 \pm 10.7$ & 0.958 \\
\hline Stay at home or in a rehabilitation unit until day 30 [days] & $156^{* * *}$ & & $1.1 \pm 4.3$ & $3.4 \pm 6.3$ & 0.022 \\
\hline
\end{tabular}

Data are presented as $\mathrm{n}(\%)$, mean \pm standard deviation.

${ }^{*} n=175$ (2 missing)

${ }^{* *} n=172$ (5 missing)

${ }^{* * *} n=156$ (only patients who reached day 30 were included)

Abbreviations: VRE vancomycin-resistant Enterococcus faecium, VSE vancomycin-susceptible E. faecium, ICU intensive care unit 
considered by multivariate logistic regression analysis, we could not outline a difference of the mortality risk between the VRE and VSE groups. In contrast, the mean SOFANon-GCS Score (from day -7 to day -1 ) was an independent and strong predictor for mortality either on day 30 or 90 post-bacteraemia onset. The relative increase of the mortality risk was $32.1 \%$ (95\% confidence interval (CI) 14.1$53.1 \%$ ) (day 30 post-BSI onset) and $17.9 \%$ (day 90 post-BSI onset), respectively, for each additional point in the SOFANon-GCS score. A similar finding was obtained for the combined endpoint, with an elevated risk for an unfavourable outcome at day 30 and 90 of 24.8 and $17.9 \%$, respectively. As already shown in the univariate analysis, even after adjustment for confounders, vancomycin resistance was not identified as an independent risk factor for mortality or unfavourable outcome after 30 (Tables 3, 4) or 90 days (Additional file 1: Table S4 and Additional file 1:Table S5).

\section{Discussion}

In this multivariate retrospective analysis on the outcome of patients with VRE- or VSE-BSI after OLT, vancomycin resistance was not identified to be a risk factor for mortality and was not associated with an increased 30- or 90-day mortality. This lack of an effect was confirmed even after adjustment for various potential confounders. Likewise, there was no association with the combined endpoint of an unfavourable outcome, which refers to death or prolonged need for ICU treatment.

To focus on this group of patients is relevant because of their notoriously high risk for VRE and it allows outcome analysis in a more homogenous cohort than in several other investigations, which included patients with broadly varying underlying diseases [24-27]. Mortality rates 30 days after
VRE-BSI and VSE-BSI ranging from 11.0 to $46.4 \%$ and 9 to $38 \%$, respectively, have been reported [24, 28-31]. Consistent with these data, mortality rates of 15.4 and $10.9 \%$ after VREand VSE-BSI were observed within the presented work. Similarly, 38.5\% of VRE and 31.2\% of VSE patients died after 90 days $(p=0.391)$.

To the best of our knowledge, three meta-analyses in this field evaluated the effect of VRE bacteraemia within the last two decades and attributed an increased risk of death to vancomycin resistance [12, 13, 32]. Two of these three meta-analyses [12, 32], and other studies that specifically focussed on liver transplant patients [10,33], included data obtained in an era before effective antibiotic treatment for VRE bacteraemia (e.g., linezolid) was broadly available. Hence, these studies are not comparable to our results. Another problem of the abovementioned meta-analyses is the inclusion of bacteraemia cases caused by different species of enterococci. This issue implicates a relevant risk of bias due to varying pathogenicity [34, 35] and virulence of E. faecium and E. faecalis and even other enterococcal species. A German study [36] reported increased in-hospital mortality in patients with E. faecium compared to E. faecalis BSI even after adjusting for underlying disease and vancomycin resistance. Similarly, even after adjustment for effective antibiotic treatment, Hayakawa et al. [37] observed impaired outcome following BSI due to vancomycinresistant E. faecium compared to vancomycin-resistant E. faecalis strains. Prematunge et al. [13] already referred to this fact in their meta-analysis, stating that many observations on VRE outcomes might be based on differences in the causative species rather than resistance itself. Analogous to our study, Yoo et al. [38] analysed

Table 3 Risk factors for death within 30 days after Enterococcus faecium bacteraemia (Cox regression; $n=177$ )

\begin{tabular}{|c|c|c|c|c|c|c|c|c|}
\hline \multirow[b]{2}{*}{ Variables } & \multirow[b]{2}{*}{$\begin{array}{l}\text { Dead } \\
n=21\end{array}$} & \multirow[b]{2}{*}{$\begin{array}{l}\text { Alive } \\
n=156\end{array}$} & \multicolumn{3}{|c|}{ Univariate } & \multicolumn{3}{|c|}{ Multivariate } \\
\hline & & & $\mathrm{HR}$ & $95 \% \mathrm{Cl}$ & $p$ & $\mathrm{HR}$ & $95 \% \mathrm{Cl}$ & $p$ \\
\hline \multicolumn{9}{|l|}{ Definitive infectious focus elimination } \\
\hline Yes & $6(28.6)$ & $40(25.6)$ & 0.85 & $0.33-2.20$ & 0.745 & 0.50 & $0.19-1.37$ & 0.181 \\
\hline No & $15(71.4)$ & $116(74.4)$ & & & & & & \\
\hline \multicolumn{9}{|l|}{ Start of adequate $A B$ Therapy within $<48 \mathrm{~h}$ post-BSI onset } \\
\hline Yes & $14(66.7)$ & $90(57.7)$ & 1.47 & $0.59-3.64$ & 0.407 & 1.03 & $0.39-2.74$ & 0.952 \\
\hline No & $7(33.3)$ & $66(42.3)$ & & & & & & \\
\hline Mean SOFA $A_{\text {Non-GCS }}$ (day-7 until day-1 before BSI onset) & $12.8 \pm 3.8$ & $6.8 \pm 4.7$ & 1.30 & $1.16-1.46$ & $<0.001$ & 1.32 & $1.14-1.53$ & $<0.001$ \\
\hline ICU stay between day-7 and BSI onset [days] & $7.6 \pm 1.2$ & $5.0 \pm 3.5$ & 1.47 & $1.08-2.00$ & 0.014 & 1.01 & $0.71-1-44$ & 0.966 \\
\hline \multicolumn{9}{|l|}{ Bacteraemia } \\
\hline VRE & $6(15.4)$ & 33 (84.6) & 1.49 & $0.58-3.84$ & 0.409 & 1.80 & $0.64-5.03$ & 0.264 \\
\hline VSE & $15(10.9)$ & $123(89.1)$ & & & & & & \\
\hline
\end{tabular}

Data are presented as $\mathrm{n}(\%)$ or mean \pm standard deviation.

Abbreviations: $H R$ hazards ratio, $C l$ confidence interval, $A B$ antibiotic therapy, $B S I$ bloodstream infection, SOFA sequential organ failure assessment, GCS Glasgow coma scale, VRE vancomycin-resistant Enterococcus faecium, VSE vancomycin-susceptible Enterococcus faecium 
Table 4 Factors associated with unfavourable outcomes 30 days after Enterococcus faecium bacteraemia (logistic regression)

\begin{tabular}{|c|c|c|c|c|c|c|c|c|}
\hline \multirow[b]{2}{*}{ Variables } & \multirow[b]{2}{*}{$\begin{array}{l}\text { Patients with favourable } \\
\text { outcome } \\
n=95\end{array}$} & \multirow[b]{2}{*}{$\begin{array}{l}\text { Patients with } \\
\text { unfavourable outcome } \\
n=80\end{array}$} & \multicolumn{3}{|c|}{ Univariate } & \multicolumn{3}{|c|}{ Multivariate } \\
\hline & & & $\mathrm{OR}$ & $95 \% \mathrm{Cl}$ & $p$ & $\mathrm{OR}$ & $95 \% \mathrm{Cl}$ & $p$ \\
\hline \multicolumn{9}{|l|}{ Definitive infectious focus elimination } \\
\hline Yes & $28(29.5)$ & $17(21.2)$ & 1.55 & $0.77-3.10$ & 0.217 & 1.92 & $0.79-4.66$ & 0.150 \\
\hline No & $67(70.5)$ & $63(78.8)$ & & & & & & \\
\hline \multicolumn{9}{|c|}{ Start of adequate $\mathrm{AB}$ Therapy within $<48 \mathrm{~h}$ post-BSI onset } \\
\hline Yes & $48(50.5)$ & $54(67.5)$ & 2.03 & $1.10-3.77$ & 0.0241 & 1.62 & $0.73-3.60$ & 0.238 \\
\hline No & $47(49.5)$ & $26(32.5)$ & & & & & & \\
\hline $\begin{array}{l}\text { Mean SOFA } A_{\text {Non-GCS }} \text { (day-7 until day-1 be- } \\
\text { fore BSI onset) }\end{array}$ & $4.8 \pm 3.8$ & $10.8 \pm 4.2$ & 1.39 & $1.26-1.53$ & $\begin{array}{l}< \\
0.001\end{array}$ & $1.2 \%$ & $1.10-1.41$ & $\begin{array}{l}< \\
0.001\end{array}$ \\
\hline $\begin{array}{l}\text { ICU stay between day-7 and BSI onset } \\
\text { [days] }\end{array}$ & $3.7 \pm 3.5$ & $7.4 \pm 1.8$ & 1.55 & $1.34-1.78$ & $\begin{array}{l}< \\
0.001\end{array}$ & 1.24 & $1.03-1.49$ & 0.023 \\
\hline \multicolumn{9}{|l|}{ Bacteraemia } \\
\hline VRE & 19 (48.7) & $20(51.3)$ & 1.33 & $0.65-2.72$ & 0.429 & 1.13 & $0.46-2.76$ & 0.795 \\
\hline VSE & 76 (55.9) & $60(44.1)$ & & & & & & \\
\hline
\end{tabular}

Favourable outcome was defined as stay at home, on the ward or in a rehabilitation centre; unfavourable outcome was defined as death or ongoing need for ICU treatment.

Data are presented as $\mathrm{n}(\%)$ or mean \pm standard deviation.

${ }^{*} n=175$ (2 missing)

Abbreviations: $O R$ odds ratio, $C l$ confidence interval, $A B$ antibiotic therapy, $B S I$ bloodstream infection, SOFA sequential organ failure assessment, GCS Glasgow coma scale, VRE vancomycin-resistant Enterococcus faecium, VSE vancomycin-susceptible Enterococcus faecium

enterococcal BSI cases strictly focussing on E. faecium; however, their number of patients was very small and information about the time to adequate antibiotic therapy was lacking.

The meta-analysis of Prematunge et al. [13], who exclusively focussed on findings after the widespread use of linezolid, also derived a higher mortality risk due to vancomycin resistance from cohort studies (hazards ratio 1.80; 95\% confidence interval 1.38-2.35). However, seven of the 12 included studies did not adjust for confounders; which is an important methodological flaw $[14,39]$. Therefore, as the authors concede, these studies could not truly figure out whether differing mortality rates mirror the effect of resistance itself or rather different underlying conditions of patients affected by VRE versus VSE bacteraemia. Corroborating this assumption, studies that adjusted for potential confounders could not identify an effect of vancomycin resistance on mortality after enterococcal bacteraemia [25, 26, 29, 40, 41]. Our study was specifically designed to elaborate the mere impact of VRE on outcomes by meticulously addressing the influence of the underlying disease severity, including previous need of ICU treatment, adequacy and timeliness of antibiotic treatment and control of the infectious focus.

Most studies, that examined the underlying severity of illness in this field, relied on scores on the day of BSI onset. This approach is problematic because values assessed on the first day of bacteraemia might already reflect the effects of the BSI rather than the patients' acute state of illness independent from and prior to bacteraemia. To circumvent this problem in our study, SOFA ${ }_{\text {Non-GCS }}$ Scores from day -7 until the day before BSI onset were collected. Multivariate analysis confirmed the overwhelming impact of the acute state of illness on the patients'outcome. Similarly, Cheah et al. [25], identified a strong association between the underlying status of the patient (as assessed by charlson comorbidity index) before enterococcal BSI and death. This appraisal is corroborated by the finding that interventions to increase arterial graft perfusion were performed more frequently in VRE patients, reflecting that a higher proportion of them were exposed to a critically compromised graft perfusion predisposing to chronic ischemic bile duct damage and a high risk of chronic hepatic infections, including liver abscess and cholangitis [42]. This was again mirrored by the distribution of infectious focuses with a broad majority of intra-abdominal sources of enterococcal bacteraemia and the corresponding increased mortality rates in this patient subgroup.

Time to the application of an effective antimicrobial substance is crucial for successful BSI treatment. Recent analyses of enterococcal blood stream infections showed worse outcome when adequate antibiotic treatment was delayed [25, 43, 44]; Zasowski et al. [44] and Vergis et al. [11] identified a breakpoint of $48 \mathrm{~h}$ after BSI onset. Several studies that supposed a worse outcome associated with VRE compared to VSE bacteraemia [27, 28, 30] adjusted for neither adequacy nor timeliness of antibiotic therapy. Therefore, this aspect was specifically addressed in the 
multivariate analysis in the present work. Definitive infectious focus elimination could only be achieved in a minority of patients in both groups mirroring the complex underlying anatomical and vascular disturbances in this selected group of patients, which is a typical scenario predisposing for enterococcal bacteraemia [45]. This aspect is another confounder that is rarely addressed in enterococcal bacteraemia studies. The finding, that the 30- and 90day mortality rates of VRE (15.4 and 38.5\%) and VSE patients (10.9 and 31.2\%) were nevertheless moderate might be explained by the fact, that measures to control the infectious source were systematically performed in all patients, even when complete eradication was reached only in the minority of them. Moreover, the moderate mortality rates might also mirror the relatively low virulence of enterococci compared to gram-negative rods or Staphylococcus aureus [46].

After carefully adjusting for confounding factors, our results suggest that not vancomycin resistance itself, but the severity of illness before E. faecium BSI onset, was associated with death and an unfavourable outcome. Given these results, one could put contact isolation policies in question. The body of evidence is steadily increasing, that -provided the compliance with standard hygiene and routine cleaning procedures is high- isolation measures due to VRE can be suspended without harm and even reduce other non-infectious adverse events $[47,48]$. International recommendations for the prevention of colonisation and infection of VRE show a wide heterogenicity and no consensus, probably due to a differing prevalence and varying structures of international health care systems $[49,50]$.

The proportion of linezolid resistant Enterococcus faecium isolates was nearly the same in the VRE- and the VSE-group (2.6 and 2.2\%, respectively). Nevertheless, vancomycin-resistance is a main-driver of linezolid use contributing to selection pressure in favour of linezolidresistant isolates. Therefore, another important part of preventive strategies are antibiotic stewardship programs.

This study has several limitations. First, it was a retrospective single-centre study. Therefore, the generalisability of our findings to other settings or patient cohorts must be considered cautiously. Second, because breakpoints for daptomycin susceptibility testing of enterococci are not provided by the European Committee on Antimicrobial Susceptibility Testing (EUCAST), the clinical isolates in this study were not routinely tested. Therefore, we have no data on potential therapeutic alternatives beyond linezolid to treat patients with VRE bacteraemia. Recent studies and meta-analyses that compared daptomycin and linezolid could not provide a definitive answer to this question, but some studies tend to favour daptomycin [51-53]. Third, we did not provide information about the genotypes of the included E. faecium strains. Researchers who focus on the influence of different genotypes state that the VanB genotype is associated with increased length of stay compared to the VanA genotype [54]. This topic requires further investigation. Fourth, immunosuppression is a potentially important factor in this population. Since we do not have individual data about the immunosuppressive medication in every patient, we were not able to consider this aspect in the multivariate analysis. This could have influenced our results and is another limitation of this study.

\section{Conclusion}

In summary, our multivariate analysis identified the mean-SOFA $\mathrm{N}_{\mathrm{Non}-\mathrm{GCS}}$ scores before BSI onset as the only strong and independent predictor of mortality. Each additional point increased the likelihood of death after 30 days by $32.1 \%$ (95\% CI $14.1-53.1 \%$ ) and also the potential for an unfavourable outcome by day 30 and 90 post-BSI. In contrast, in an era of broadly available effective treatment, vancomycin resistance had no impact on mortality or a poor outcome in patients with E. faecium BSI after OLT.

\section{Supplementary information}

Supplementary information accompanies this paper at https://doi.org/10. 1186/s13756-020-0683-3.

\section{Additional file 1: Table S1. Polymicrobial blood stream infections and identified Co-pathogens in study subjects. Table S2. Antibiotic therapy used to treat the study subjects Table S3. Interventions from blood stream infec- tion (BSI) until day 30 Table S4. Risk factors for death within 90 days after Enterococcus faecium bacteraemia (Cox regression). Table S5. Factors associ- ated with unfavourable outcomes 90 days after Enterococcus faecium bacter- aemia (logistic regression). Table S6. Source of infection and related outcome in patients with vancomycin-resistant Enterococcus faecium (VRE) \\ Table S7. Source of infection and related outcome in patients with vancomycin-susceptible E. faecium (VSE) bacteraemia.}

\section{Abbreviations \\ BDA: Biliodigestive anastomosis; BSI: Blood stream infections; Cl: Confidence interval; ERCP: Endoscopic retrograde cholangiopancreatography; EUCAST: European Committee on Antimicrobial Susceptibility Testing; HR: Hazard ratio; ICU: Intensive care unit; MDRO: Multidrug-resistant organisms; MELD: Model of end stage liver disease; OLT: Orthotopic liver transplantation; PCR: Polymerase chain reaction; PTCD: Percutaneous transhepatic cholangiodrainage; SOFA: Sequential organ failure assessment score; VRE: Vancomycin-resistant enterococci; VSE: Vancomycin-susceptible enterococci}

\section{Acknowledgements \\ We do not have any Acknowledgments.}

\section{Authors' contributions}

SD was a major contributor in writing of the manuscript, ML captured patient data, SZ interpreted and analysed microbiological samples, DCR substantively revised the manuscript, MM helped in interpreting data, TB conducted and performed statistical analysis, AM helped in interpreting data, KHW helped in interpreting data, MAW substantively revised the manuscript, TB concepted and designed the study, $\mathrm{AH}$ concepted and designed the study. All authors read and approved the final manuscript.

Funding

The present research was not funded institutionally or by anyone else. 


\section{Availability of data and materials}

All data generated or analysed during this study are included in this published article [and its supplementary information files] Original data are available from the corresponding author upon reasonable request.

\section{Ethics approval and consent to participate}

Ethics approval by the local ethics committee of the Medical Faculty of the University of Heidelberg, Germany (S-407/2017) and was registered at the German clinical trials register (DRKS-ID: DRKS00013285).

\section{Consent for publication}

Not applicable.

\section{Competing interests}

Karl-Heinz Weiss advises for Alexion, Univar, GMP-O, Ipsen, Chiesi, Novartis. Karl-Heinz Weiss is on the speaker's bureau of Pfizer, Abbvie, Eisai. Karl-Heinz Weiss received grant support (to the institution) from AstraZenica, QED, Novartis, Univar, Alexion and GMP-O. Markus Weigand is on the advisory boards from MSD, Gilead, Pfizer, Shionogi. Thorsten Brenner received research funding from German Research Foundation (DFG), Heidelberg Foundation of Surgery, Dietmar Hopp Foundation. Thorsten Brenner received honoraria for lectures and advisory boards from: Biotest AG, Baxter Deutschland $\mathrm{GmbH}$, Schöchl medical education $\mathrm{GmbH}$, Boehringer Ingelheim Pharma GmbH, CSL Behring GmbH, Astellas Pharma GmbH, B. Braun Melsungen AG, MSD Sharp \& Dohme GmbH. Alexandra Heininger received speaker honorary by MSD and Pfizer. Simon Dubler, Martin Lenz, Stefan Zimmermann, Daniel Richter, Arianeb Mehrabi, Markus Mieth and Thomas Brucker declare that they have no competing interests.

\section{Author details}

'Department of Anaesthesiology, Heidelberg University Hospital, Im Neuenheimer Feld 110, D-69120 Heidelberg, Germany. ${ }^{2}$ Department of Anaesthesiology, Intensive Care and Emergency Medicine, Asklepios Clinics Hamburg, AK Wandsbek, Hamburg, Germany. ${ }^{3}$ Department of Infectious Diseases, Medical Microbiology and Hygiene, Division Bacteriology, Heidelberg University Hospital, Heidelberg, Germany. ${ }^{4}$ Department of Internal Medicine, Heidelberg University Hospital, Heidelberg, Germany. ${ }^{5}$ Department of Visceral and Transplant Surgery, Heidelberg University Hospital, Heidelberg, Germany. ${ }^{6}$ Institute for Medical Biometry and Informatics, University of Heidelberg, Heidelberg, Germany. ${ }^{7}$ Division Hospital and Environmental Hygiene Department of Infectious Diseases, Medical Microbiology and Hygiene, Heidelberg University Hospital, Heidelberg, Germany.

Received: 25 November 2019 Accepted: 22 January 2020

Published online: 31 January 2020

\section{References}

1. Sievert DM, Ricks P, Edwards JR, Schneider A, Patel J, Srinivasan A, et al. Antimicrobial-resistant pathogens associated with healthcare-associated infections: summary of data reported to the National Healthcare Safety Network at the Centers for Disease Control and Prevention, 2009-2010. Infect Control Hosp Epidemiol. 2013;34(1):1-14.

2. Schwab F, Geffers C, Behnke M, Gastmeier P. ICU mortality following ICUacquired primary bloodstream infections according to the type of pathogen: a prospective cohort study in 937 Germany ICUs (2006-2015). PLoS One. 2018;13(3):e0194210.

3. Tacconelli E, Magrini N. GLOBAL PRIORITY LIST OF ANTIBIOTIC-RESISTANT BACTERIA TO GUIDE RESEARCH, DISCOVERY, AND DEVELOPMENT OF NEW ANTIBIOTICS. World Health Organization (WHO). 2017:7.

4. Wisplinghoff $H$, Bischoff $T$, Tallent SM, Seifert H, Wenzel RP, Edmond MB Nosocomial bloodstream infections in US hospitals: analysis of 24,179 cases from a prospective nationwide surveillance study. Clin Infect Dis. 2004;39(3):309-17.

5. (ECDC) ECfDPaC. Annual epidemiological report for 2016 - Healthcareassociated infections acquired in intensivecare units. ECDC Annual epidemiological report for 2016. 2018.

6. Noskin GA, Peterson LR, Warren JR. Enterococcus faecium and Enterococcus faecalis bacteremia: acquisition and outcome. Clin Infect Dis. 1995;20(2):296-301.

7. Pinholt M, Ostergaard C, Arpi M, Bruun NE, Schonheyder HC, Gradel KO, et al. Incidence, clinical characteristics and 30-day mortality of enterococcal bacteraemia in Denmark 2006-2009: a population-based cohort study. Clin Microbiol Infect. 2014:20(2):145-51.

8. Hidron Al, Edwards JR, Patel J, Horan TC, Sievert DM, Pollock DA, et al. NHSN annual update: antimicrobial-resistant pathogens associated with healthcare-associated infections: annual summary of data reported to the National Healthcare Safety Network at the Centers for Disease Control and Prevention, 2006-2007. Infect Control Hosp Epidemiol. 2008;29(11):996-1011.

9. Remschmidt C, Schneider S, Meyer E, Schroeren-Boersch B, Gastmeier P, Schwab F. Surveillance of antibiotic use and resistance in intensive care units (SARI). Dtsch Arztebl Int. 2017;114(50):858-65.

10. Linden PK, Pasculle AW, Manez R, Kramer DJ, Fung JJ, Pinna AD, et al. Differences in outcomes for patients with bacteremia due to vancomycinresistant Enterococcus faecium or vancomycin-susceptible E. faecium. Clin Infect Dis. 1996;22(4):663-70.

11. Vergis EN, Hayden MK, Chow JW, Snydman DR, Zervos MJ, Linden PK, et al. Determinants of vancomycin resistance and mortality rates in enterococcal bacteremia. A prospective multicenter study. Ann Intern Med. 2001;135(7):484-92

12. DiazGranados CA, Zimmer SM, Klein M, Jernigan JA. Comparison of mortality associated with vancomycin-resistant and vancomycin-susceptible enterococcal bloodstream infections: a meta-analysis. Clin Infect Dis. 2005; 41(3):327-33.

13. Prematunge C, MacDougall C, Johnstone J, Adomako K, Lam F, Robertson J, et al. VRE and VSE bacteremia outcomes in the era of effective VRE therapy: a systematic review and meta-analysis. Infect Control Hosp Epidemiol. 2016; 37(1):26-35.

14. Blot S, Depuydt P, Vandewoude K, De Bacquer D. Measuring the impact of multidrug resistance in nosocomial infection. Curr Opin Infect Dis. 2007; 20(4):391-6.

15. Timsit JF, Sonneville R, Kalil AC, Bassetti M, Ferrer R, Jaber S, et al. Diagnostic and therapeutic approach to infectious diseases in solid organ transplant recipients. Intensive Care Med. 2019;45(5):573-91.

16. Sganga G, Spanu T, Bianco G, Fiori B, Nure E, Pepe G, et al. Bacterial bloodstream infections in liver transplantation: etiologic agents and antimicrobial susceptibility profiles. Transplant Proc. 2012:44(7):1973-6.

17. Nellore A, Huprikar S, AICo P. Vancomycin-resistant Enterococcus in solid organ transplant recipients: Guidelines from the American Society of Transplantation Infectious Diseases Community of Practice. Clin Transplant. 2019;33(9):e13549.

18. Vincent $\mathrm{J}$, de Mendonca A, Cantraine F, Moreno R, Takala J, Suter PM, et al. Use of the SOFA score to assess the incidence of organ dysfunction/failure in intensive care units: results of a multicenter, prospective study. Working group on "sepsis-related problems" of the European Society of Intensive Care Medicine. Crit Care Med. 1998:26(11):1793-800.

19. Vasilevskis EE, Pandharipande PP, Graves AJ, Shintani A, Tsuruta R, Ely EW, et al. Validity of a modified sequential organ failure assessment score using the Richmond agitation-sedation scale. Crit Care Med. 2016;44(1):138-46.

20. Viglino D, Maignan M, Debaty G. A modified sequential organ failure assessment score using the Richmond agitation-sedation scale in critically ill patients. J Thorac Dis. 2016;8(3):311-3.

21. Klein S, Zimmermann S, Kohler C, Mischnik A, Alle W, Bode KA. Integration of matrix-assisted laser desorption/ionization time-of-flight mass spectrometry in blood culture diagnostics: a fast and effective approach. J Med Microbiol. 2012;61(Pt 3):323-31.

22. Montravers P, Blot S, Dimopoulos G, Eckmann C, Eggimann P, Guirao X, et al. Therapeutic management of peritonitis: a comprehensive guide for intensivists. Intensive Care Med. 2016;42(8):1234-47.

23. Hecker A, Reichert M, Reuss CJ, Schmoch T, Riedel JG, Schneck E, et al. Intraabdominal sepsis: new definitions and current clinical standards. Langenbeck's Arch Surg. 2019;404(3):257-71.

24. Han SH, Chin BS, Lee HS, Jeong SJ, Choi HK, Kim CO, et al. Vancomycinresistant enterococci bacteremia: risk factors for mortality and influence of antimicrobial therapy on clinical outcome. J Inf Secur. 2009;58(3):182-90.

25. Cheah AL, Spelman T, Liew D, Peel T, Howden BP, Spelman D, et al. Enterococcal bacteraemia: factors influencing mortality, length of stay and costs of hospitalization. Clin Microbiol Infect. 2013;19(4):E181-9.

26. Billington EO, Phang $\mathrm{SH}$, Gregson DB, Pitout JD, Ross T, Church DL, et al. Incidence, risk factors, and outcomes for Enterococcus spp. blood stream infections: a population-based study. Int J Infect Dis. 2014;26:76-82.

27. da Silva NS, Muniz VD, Estofolete CF, Furtado GH, Rubio FG. Identification of temporal clusters and risk factors of bacteremia by nosocomial vancomycinresistant enterococci. Am J Infect Control. 2014;42(4):389-92. 
28. Vydra J, Shanley RM, George I, Ustun C, Smith AR, Weisdorf DJ, et al Enterococcal bacteremia is associated with increased risk of mortality in recipients of allogeneic hematopoietic stem cell transplantation. Clin Infect Dis. 2012;55(6):764-70.

29. Cho SY, Lee DG, Choi SM, Kwon JC, Kim SH, Choi JK, et al. Impact of vancomycin resistance on mortality in neutropenic patients with enterococcal bloodstream infection: a retrospective study. BMC Infect Dis. 2013;13:504

30. Weber S, Hogardt M, Reinheimer C, Wichelhaus TA, Kempf VAJ, Kessel J, et al. Bloodstream infections with vancomycin-resistant enterococci are associated with a decreased survival in patients with hematological diseases. Ann Hematol. 2019;98(3):763-73.

31. Mikulska M, Del Bono V, Raiola AM, Signori A, Prinapori R, Ghiso A, et al. Enterococcal bloodstream infection after hematopoietic stem cell transplant: experience of a center with a low prevalence of vancomycinresistant enterococci. Clin Infect Dis. 2012;55(12):1744.

32. Salgado CD, Farr BM. Outcomes associated with vancomycin-resistant enterococci: a meta-analysis. Infect Control Hosp Epidemiol. 2003;24(9):690-8.

33. Gearhart M, Martin J, Rudich S, Thomas M, Wetzel D, Solomkin J, et al. Consequences of vancomycin-resistant Enterococcus in liver transplant recipients: a matched control study. Clin Transpl. 2005;19(6):711-6.

34. Sava IG, Heikens E, Huebner J. Pathogenesis and immunity in enterococcal infections. Clin Microbiol Infect. 2010;16(6):533-40.

35. Arias CA, Murray BE. The rise of the Enterococcus: beyond vancomycin resistance. Nat Rev Microbiol. 2012;10(4):266-78.

36. Kramer TS, Remschmidt C, Werner S, Behnke M, Schwab F, Werner G, et al. The importance of adjusting for enterococcus species when assessing the burden of vancomycin resistance: a cohort study including over 1000 cases of enterococcal bloodstream infections. Antimicrob Resist Infect Control. 2018;7:133.

37. Hayakawa K, Marchaim D, Martin ET, Tiwari N, Yousuf A, Sunkara B, et al. Comparison of the clinical characteristics and outcomes associated with vancomycin-resistant Enterococcus faecalis and vancomycin-resistant $\mathrm{E}$. faecium bacteremia. Antimicrob Agents Chemother. 2012;56(5):2452-8.

38. Yoo JH, Lee DG, Choi SM, Choi JH, Shin WS, Kim M, et al. Vancomycin-resistant enterococcal bacteremia in a hematology unit: molecular epidemiology and analysis of clinical course. J Korean Med Sci. 2005;20(2):169-76.

39. Schwaber MJ, Carmeli Y. Antimicrobial resistance and patient outcomes: the hazards of adjustment. Crit Care. 2006;10(5):164.

40. Marschall J, Piccirillo ML, Fraser VJ, Doherty JA, Warren DK. Catheter removal versus retention in the management of catheter-associated enterococcal bloodstream infections. Can J Infect Dis Med Microbiol. 2013;24(3):e83-7.

41. Bar K, Wisplinghoff H, Wenzel RP, Bearman GM, Edmond MB. Systemic inflammatory response syndrome in adult patients with nosocomial bloodstream infections due to enterococci. BMC Infect Dis. 2006;6:145.

42. Haidar G, Green M. Intra-abdominal infections in solid organ transplant recipients: Guidelines from the American Society of Transplantation Infectious Diseases Community of Practice. Clinical Transplantation. 2019; 33(9):e13595. https://doi.org/10.1111/ctr.13595

43. Suppli M, Aabenhus R, Harboe ZB, Andersen LP, Tvede M, Jensen JU. Mortality in enterococcal bloodstream infections increases with inappropriate antimicrobial therapy. Clin Microbiol Infect. 2011;17(7):1078-83.

44. Zasowski EJ, Claeys KC, Lagnf AM, Davis SL, Rybak MJ. Time is of the essence: the impact of delayed antibiotic therapy on patient outcomes in hospital-onset Enterococcal bloodstream infections. Clin Infect Dis. 2016; 62(10):1242-50.

45. Montravers P, Dufour G, Guglielminotti J, Desmard M, Muller C, Houissa H, et al. Dynamic changes of microbial flora and therapeutic consequences in persistent peritonitis. Crit Care. 2015;19:70.

46. Frickmann $H$, Koller K, Veil I, Weise M, Ludyga A, Schwarz NG, et al. On the role of enterococci in the bloodstream: results of a single-center, retrospective, observational study at a German University hospital. Eur J Microbiol Immunol (Bp). 2017;7(4):284-95.

47. Bearman G, Abbas S, Masroor N, Sanogo K, Vanhoozer G, Cooper K, et al. Impact of discontinuing contact precautions for methicillin-resistant Staphylococcus aureus and Vancomycin-resistant Enterococcus: an interrupted time series analysis. Infect Control Hosp Epidemiol. 2018:39(6):676-82.

48. Martin EM, Bryant B, Grogan TR, Rubin ZA, Russell DL, Elashoff D, et al. Noninfectious hospital adverse events decline after elimination of contact precautions for MRSA and VRE. Infect Control Hosp Epidemiol. 2018;39(7):788-96.
49. Cookson BD, Macrae MB, Barrett SP, Brown DF, Chadwick C, French GL, et al. Guidelines for the control of glycopeptide-resistant enterococci in hospitals. J Hosp Infect. 2006;62(1):6-21.

50. Government of South Australia (SA Health) (2017) Management of Patients with Vancomycin-resistant enterococci (VRE) clinical guideline.http:// wwwsahealthsagovau/wps/wcm/connect/public+content/sa+health+ internet/resources/policies/vancomycin-resistant+enterococci+vre+patient+ management+clinical+guideline 2017; Last Access: 3rd January 2020.

51. Britt NS, Potter EM, Patel N, Steed ME. Comparison of the effectiveness and safety of linezolid and Daptomycin in Vancomycin-resistant Enterococcal bloodstream infection: a National Cohort Study of veterans affairs patients. Clin Infect Dis. 2015;61(6):871-8.

52. Chuang YC, Lin HY, Chen PY, Lin CY, Chen YC, Wang JT, et al. Survival of patients with Vancomycin-resistant Enterococcus faecium bacteremia treated with conventional or high doses of Daptomycin or linezolid is associated with the rate of bacterial clearance. Crit Care Med. 2018;46(10):1634-42.

53. Kanjilal S, Kalil AC, Klompas M. What is the best treatment for Vancomycinresistant Enterococcal bloodstream infections? Crit Care Med. 2018;46(10):1700-3.

54. Cheah AL, Peel T, Howden BP, Spelman D, Grayson ML, Nation RL, et al. Case-case-control study on factors associated with vanB vancomycinresistant and vancomycin-susceptible enterococcal bacteraemia. BMC Infect Dis. 2014;14:353.

\section{Publisher's Note}

Springer Nature remains neutral with regard to jurisdictional claims in published maps and institutional affiliations.

\section{Ready to submit your research? Choose BMC and benefit from:}

- fast, convenient online submission

- thorough peer review by experienced researchers in your field

- rapid publication on acceptance

- support for research data, including large and complex data types

- gold Open Access which fosters wider collaboration and increased citations

- maximum visibility for your research: over $100 \mathrm{M}$ website views per year

At $\mathrm{BMC}$, research is always in progress.

Learn more biomedcentral.com/submissions 